\title{
A EDUCAÇÃO PROFISSIONAL EM SAÚDE NO CENTRO DE FORMAÇÃO DE PESSOAL PARA OS SERVIÇOS DE SAÚDE DR. MANOEL DA COSTA SOUZA, DE 1997 A 2015
}

\author{
Thais Paulo Teixeira Costa; Janete Lima de Castro \\ Núcleo de Estudos em Saúde Coletiva - Universidade Federal do Rio Grande do Norte \\ DOI: 10.15628/rbept.2018.7334
}

Artigo submetido em maio/2018 e aceito em ago/2018

\begin{abstract}
RESUMO
Este artigo tem o objetivo de apresentar os cursos desenvolvidos pelo Centro de Formação de Pessoal para os Serviços de Saúde Dr. Manoel da Costa Souza (CEFOPE/RN), espaço responsável pela formação dos trabalhadores de nível médio e elementar que atuam no Sistema Único de Saúde, entre 1997 e 2015. A pesquisa teve como fonte de dados as atas escolares disponibilizadas pela Secretaria Escolar do CEFOPE/RN, analisadas in loco entre abril e junho de 2017. A análise realizada mostra que a trajetória do CEFOPE/RN se constituiu dentro de um espaço de fortalecimento para os trabalhadores atuantes na área da saúde, e os cursos ofertados demonstram a preocupação com a melhoria da qualidade dos serviços de saúde pública do país, uma vez que grande parte dos cursos ofertados está coerente com programas nacionais vinculados ao Ministério da Saúde. Assim, esta pesquisa sugere novos mapeamentos na referida instituição, no intuito de continuar contribuindo com sua trajetória e com a compreensão acerca da formação destinada aos trabalhadores de nível médio em saúde.
\end{abstract}

Palavras-Chave: Educação Profissional em Saúde Pública. Sistema Único de Saúde. Educação Permanente em Saúde.

\section{THE PROFESSIONAL HEALTH EDUCATION IN THE DR. MANOEL DA COSTA STAFF TRAINING CENTER FOR THE HEALTH SERVICES FROM 1997 TO 2015}

\begin{abstract}
The paper is focused on the courses developed by the Staff Training Center for Health Services Dr. Manoel da Costa Souza (CEFOPE / RN), responsible for the middle and elementary level staff training for the Unified Health System during the period from 1997 to 2015. In order to understand its activities betwenn 1997 and 2015, documents such as school records (1997 - 2015) were analized. The research was carried out at CEFOPE/RN School Secretariat sector from April to June, 2017. The analysis shows the CEFOPE/RN trajectory was constituted within a space of strengthening for the health area workers and the offered courses demonstrate the concern with the improvement of the country's public health services quality, since most of the courses offered are consistent with national programs linked to the country's Ministry of Health. However, this research intends to carry us out for new mappings in the institution and continuing to contribute to its trajectory and to the understanding of the middle level workers training for health.
\end{abstract}

Keywords: Professional Education in Public Health. Health Unic System. Permanent Education in Health. 


\section{INTRODUÇÃO}

Este artigo tem o objetivo de apresentar os cursos desenvolvidos pelo Centro de Formação de Pessoal para os Serviços de Saúde Dr. Manoel da Costa Souza (CEFOPE/RN), espaço responsável pela formação dos trabalhadores de nível médio e elementar que atuam no Sistema Único de Saúde (SUS), entre1997 e 2015.

Este Centro, sendo uma das primeiras escolas técnicas de saúde pública do país, foi criado em 1984 e integra a Rede de Escolas Técnicas do Sistema Único de Saúde (RETSUS). Estudo realizado por Germano (1996) investigou a trajetória do CEFOPE/RN no período de 1984 a 1996. Então, a fim de contribuir para o registro da história do referido Centro de Formação, esta pesquisa o toma como referência, dando continuidade à proposta de conhecer e dialogar sobre a história do CEFOPE/RN no período de 1997 a 2015.

A justificativa deste estudo se dá devido à necessidade de acompanhar a formação de quem faz o serviço de saúde, visando contribuir com o fortalecimento da imagem institucional do CEFOPE/RN, a partir da socialização do trabalho desenvolvido por esta instituição, e com a Educação Profissional em Saúde no Rio Grande do Norte.

A Educação Profissional é compreendida como uma modalidade de ensino que se dedica à formação e qualificação dos trabalhadores de nível médio e, no caso da saúde, especificamente, está direcionada aos que atuam ou atuarão nos serviços de saúde (PEREIRA; RAMOS, 2006). Assim, enquanto área da Educação que pressupõe o diálogo da relação entre trabalho e educação, configura-se como uma forma de educar para o trabalho com os cuidados necessários à vida humana.

No Brasil, a Educação Profissional, inicialmente, foi destinada aos trabalhadores que se encontravam fora dos padrões, em termos de condições econômicas, aceitos como normais pela sociedade (MOURA, 2007). Seus marcos históricos têm, assim, caráter reducionista, o que limita a formação dos sujeitos ao emprego. Entretanto, identifica-se que, ao articular o trabalho como princípio educativo, a Educação Profissional possibilita a mediação entre o trabalho técnico e o trabalho intelectual, característica que ajuda a romper com a divisão técnica do trabalho entre os que fazem e aqueles que pensam.

Os trabalhadores de nível médio, em sua maioria, são de uma classe social oriunda predominantemente das camadas economicamente menos favorecidas e, muitas vezes, enfrentam uma formação baseada no tecnicismo e na repetição do trabalho. Isto implica em uma desvalorização frente aos demais trabalhadores, como os doutores e diplomados, os quais apresentam imagens instituídas como superiores, cultuadas pela sociedade. Desta forma, esses trabalhadores de nível médio enfrentam desafios tanto na formação 
recebida quanto no espaço de trabalho, sendo hierarquicamente subordinado nas relações de trabalho.

Assim sendo, formar trabalhadores técnicos requer ir além da formação reprodutivista. Trata-se de discutir a formação desses sujeitos objetivando sua emancipação humana em articulação com a realidade, de maneira que o trabalho seja compreendido para além da produção capitalista, visto que estão inseridos em um espaço de trabalho que não atende às prerrogativas de lucro do mercado capitalista.

Observa-se que a implementação do SUS, descrita na Lei no 8.080 de 1990, propôs um novo direcionamento à formação dos trabalhadores da saúde, conforme sua organização. Novos cenários e possibilidades foram desenvolvidos com o modelo de criação que tornou necessárias atividades de formação que tivessem como referência os princípios éticos da universalidade, da equidade e da integralidade, para garantir à população o acesso igualitário às ações e serviços de promoção, proteção e recuperação da saúde.

Entretanto, passados trinta anos e inúmeras ações que pretendiam aproximar as áreas da educação e da saúde, "a formação dos trabalhadores da saúde não se orienta pela leitura das necessidades sociais em saúde" (BRASIL, 2001, p. 42). Esse cenário desarticulado entre as necessidades sociais e a formação dos trabalhadores para atuar no SUS foi influenciado pela grande oferta de cursos da área da saúde em instituições públicas e privadas de ensino, com currículos fechados e pouco comprometidos com o fortalecimento das políticas públicas de saúde (SILVA; SANTANA, 2015).

Neste sentido, tornam-se necessários espaços formativos que dialoguem com os trabalhadores e os auxiliem na construção de estratégias transformadoras dos seus espaços de atuação. É oportuno refletir que as ETSUS possuem este papel formativo e, para isso, é necessário compreender sua trajetória histórica.

As ETSUS são instituições potencializadoras do debate entre a formação e o ambiente de trabalho. Podem ser consideradas como um espaço que busca a transformação social através de sua capilaridade, ao atingir municípios de difícil acesso com um ensino voltado para uma formação cidadã. Ressaltamos, também, sua historicidade como espaço resultante de lutas por reconhecimento e valorização dos trabalhadores (BRASIL, 2009).

Durante a investigação no CEFOPE/RN, surgiu o seguinte questionamento: Quais os cursos desenvolvidos pelo CEFOPE/RN nos últimos 18 anos? Estavam articulados a que propostas? Assim, ao longo deste trabalho, serão apresentadas respostas a estes questionamentos. 


\title{
2 METODOLOGIA
}

Considera-se que o cenário de atuação desses trabalhadores é extremamente complexo e mesmo inserido na Constituição Federal de 1988, - SUS ainda está em processo de consolidação em decorrência dos constantes cortes que acompanham toda a sua trajetória. Assim, os trabalhadores inseridos neste sistema convivem com enormes desafios, envolvendo o reconhecimento das atividades desenvolvidas, como também as questões salariais.

Desta forma, para compreender a realidade, é necessário investigar e analisar os motivos pelos quais determinadas circunstâncias ocorrem. Assim, com o intuito de compreender o papel do CEFOPE/RN, uma das instituições que ofertam Educação Profissional em Saúde no Rio Grande Norte, fez-se necessária uma aproximação com o materialismo histórico-dialético, conforme aborda Frigotto (1994):

\begin{abstract}
No processo dialético de conhecimento da realidade, o que importa fundamentalmente não é a crítica pela crítica, o conhecimento pelo conhecimento, mas a crítica e o conhecimento crítico para uma prática que altere e transforme a realidade anterior no plano do conhecimento e no plano histórico-social (FRIGOTTO, 1994, p. 81).
\end{abstract}

Para atingir o objetivo proposto, foram utilizadas como referências metodológicas e procedimentais Minayo (2013) e Ghedin e Franco (2011). Segundo Minayo (2013), uma pesquisa qualitativa pode ser considerada como aquela capaz de incorporar significado e intencionalidade. Deste modo, pretende-se compreender os caminhos históricos e sociais da instituição em foco, com um enquadramento qualitativo.

Ghedin e Franco (2011) salientam que o método só existe à medida que um pesquisador questiona e reflete sobre o seu objeto de estudo. Logo, aqui, buscou-se refletir sobre o sentido histórico e social do CEFOPE/RN, em sua dinâmica de ações direcionadas à Educação Profissional em Saúde.

Para tanto, foi realizada uma pesquisa documental, de abordagem qualitativa, a fim de identificar os cursos ofertados no CEFOPE/RN, no período de 1997 a 2015, por meio das atas escolares do período em estudo. A pesquisa foi realizada no CEFOPE/RN, especificamente no setor da Secretaria Escolar, no período de abril a junho de 2017.

Para a pesquisa in loco, foi realizado um contato prévio com a coordenação do CEFOPE/RN, a qual disponibilizou os documentos para a realização da análise. Assim, foram analisadas todas as atas escolares impressas e assinadas pelos diretores da Escola no período supracitado. Destaca-se que a escolha das atas ocorreu por ser o documento que possuía o maior número de registros sobre os cursos ofertados, respaldados pelas assinaturas dos diretores. $\mathrm{Na}$ pesquisa, identificou-se uma falta de padronização dos documentos, motivo pelo qual, na coleta de dados, não se registrou o ano de início dos cursos, apenas o seu período de conclusão. 


\section{RESULTADOS E DISCUSSOES}

A relação entre trabalho e educação possibilita analisar dinâmicas sociais, econômicas e políticas na sociedade envoltas em regulamentos do Estado que incidem diretamente sobre elas. Assim, compreender esta relação contribui para a análise dos desafios históricos em que as instituições encontram-se inseridas.

O CEFOPE/RN pode ser identificado como um espaço que contribui para a formação de trabalhadores do SUS, com o desafio de ofertar uma formação crítica, para além de um modelo de trabalho estruturado em demandas de mercado. Em especial, por ter como base a compreensão de que o trabalho em saúde se impõe para além do aumento da produtividade, economicidade e o instrumentalismo desse mercado, trata-se de um trabalho que requer atenção e cuidado com a vida humana.

Sendo assim, compartilhar a história do CEFOPE/RN como Escola de Educação Profissional em Saúde, instituição criada pela necessidade de estruturação do serviço de saúde e que defende uma formação adequada ao projeto de saúde pública, vinculada às necessidades objetivas e subjetivas dos usuários, é mostrar reconhecimento por esse serviço. Esta tarefa, evidentemente, torna seu espaço ainda mais desafiador.

É válido destacar que as ETSUS não foram escolas pensadas de maneira substitutiva às Escolas Técnicas existentes no Brasil. Estas Escolas acompanham e formam os trabalhadores da área da saúde, pois foram idealizadas para dar centralidade à formação destes estudantes, atendendo às suas necessidades de trabalho. Portanto, as ETSUS foram institucionalizadas para um público-alvo específico de trabalhadores do SUS, os quais se encontram no serviço. Assim, a escola precisava ser flexível para atender o perfil do indivíduo e, descentralizada, para alcançar os que atuam em diversos espaços geográficos, cumprindo com o preconizado pelo SUS, que passa a organizar-se tendo como referência uma rede regionalizada e hierarquizada de serviços de saúde (BRASIL, 2017).

A regionalização dos serviços representa um dos princípios organizativos do SUS e possibilita sua organização a partir de um dado território, buscando ofertar aos usuários o atendimento integral. Os cursos que serão aqui apresentados (Quadro 01) foram ofertados para os trabalhadores inseridos nos diversos serviços da rede de atenção à saúde do estado do Rio Grande do Norte. 


\begin{tabular}{|c|c|}
\hline $\begin{array}{l}\text { Ano de conclusão } \\
\text { do curso }\end{array}$ & Cursos concluídos \\
\hline \multirow[t]{4}{*}{1997} & Auxiliar de enfermagem \\
\hline & Auxiliar de nutrição e dietética \\
\hline & Atendente de consultório dentário \\
\hline & Auxiliar de patologia clínica \\
\hline \multirow[t]{3}{*}{1998} & Auxiliar de enfermagem \\
\hline & Atendente de consultório dentário \\
\hline & Auxiliar de patologia clínica \\
\hline \multirow[t]{2}{*}{1999} & Auxiliar de enfermagem \\
\hline & Técnico em hemoterapia \\
\hline 2000 & Auxiliar de enfermagem \\
\hline 2001 & Auxiliar de enfermagem \\
\hline 2002 & Qualificação profissional de auxiliar de enfermagem \\
\hline \multirow{9}{*}{2003} & Qualificação profissional de auxiliar de enfermagem \\
\hline & Qualificação profissional de auxiliar de enfermagem \\
\hline & Iniciação tecnológica em biodiagnóstico \\
\hline & Imunohematologia \\
\hline & Biossegurança \\
\hline & Educação em saúde \\
\hline & Atualização em registro de saúde \\
\hline & Terapia transfusional \\
\hline & Atenção ao idoso \\
\hline \multirow[t]{2}{*}{2004} & Atenção ao idoso \\
\hline & Técnico em enfermagem \\
\hline \multirow[t]{6}{*}{2005} & Qualificação profissional de auxiliar de enfermagem \\
\hline & Técnico de enfermagem \\
\hline & Técnico de hemoterapia \\
\hline & Atendente de consultório dentário \\
\hline & Técnico em higiene dental \\
\hline & Qualificação do agente comunitário de saúde \\
\hline 2006 & Qualificação do agente comunitário de saúde \\
\hline 2007 & Técnico de nível médio em gestão dos serviços de saúde \\
\hline 2008 & Técnico de nível médio em nutrição e dietética \\
\hline 2009 & Cuidador da pessoa idosa com dependência \\
\hline \multirow{7}{*}{2010} & $\begin{array}{c}\text { As ações de prevenção e controle de agravos obstetrícios e neonatais } \\
\text { na maternidade }\end{array}$ \\
\hline & $\begin{array}{c}\text { Qualificação para redução da morte materna e infantil nas ações de } \\
\text { enfermagem da atenção básica }\end{array}$ \\
\hline & $\begin{array}{l}\text { Cuidados à mulher no ciclo gravídico-puerperal e ao recém-nascido na } \\
\text { atenção básica- }\end{array}$ \\
\hline & $\begin{array}{l}\text { Ações de prevenção e controle de agravos obstetrícios e neonatais na } \\
\text { maternidade }\end{array}$ \\
\hline & $\begin{array}{c}\text { Qualificação para redução da morte materna e infantil nas ações de } \\
\text { enfermagem da atenção básica }\end{array}$ \\
\hline & Qualificação para cuidador da pessoa idosa com dependência \\
\hline & Capacitação do agente comunitário de saúde (RMI) \\
\hline 2011 & Técnico de nível médio em saúde bucal \\
\hline 2012 & Não foi registrada conclusão de curso neste ano \\
\hline 2013 & Capacitação do agente comunitário de saúde \\
\hline 2014 & Não foi registrada conclusão de curso neste ano \\
\hline \multirow[t]{3}{*}{2015} & Técnico de nível médio em radiologia \\
\hline & Técnico de nível médio em vigilância em saúde \\
\hline & Capacitação do agente comunitário de saúde \\
\hline
\end{tabular}

Quadro 01: Cursos ofertados no CEFOPE/RN, de acordo com as atas escolares, no período de 1997 a 2015.

Fonte: CEFOPE/RN, elaborado pela autora deste trabalho (2017).

Vol. 2 (2018) 
Pensando na melhoria dos processos formativos para trabalhadores da saúde atuantes em um serviço público de saúde, o Quadro 01 reforça a importância da educação profissional para atender àqueles cujo trabalho se materializa em espaços de saúde. $\mathrm{Na}$ análise, serão discutidos cursos que estavam inseridos em projetos Ministeriais.

A presença do grande quantitativo de cursos de enfermagem segue uma tendência nacional de qualificação da força de trabalho da área, expressa pelo desenvolvimento do Projeto de Profissionalização dos Trabalhadores da Área da Enfermagem (PROFAE), na tentativa de modificar a ausência histórica de cursos de formação na referida área para os trabalhadores inseridos nos serviços de saúde.

Nesse sentido, de acordo com os propósitos de estruturação das ETSUS, desenvolvidos pelo PROFAE, identifica-se que houve a organização estrutural do CEFOPE/RN, seguindo a tendência das modificações dos modelos pedagógicos nas demais ETSUS (BARRETTO, 2010). De acordo com Pereira (2002), a Escola Izabel dos Santos, também integrante da ETSUS, teve este projeto como principal atividade no ano de 2002.

$\mathrm{Na}$ análise dos cursos desenvolvidos pelo CEFOPE/RN, destaca-se também o Curso de Qualificação do Agente Comunitário de Saúde, desenvolvido nos anos de 2005, 2006 e 2015. O Agente Comunitário de Saúde (ACS) integra ações da Atenção Básica em Saúde (AB), na Equipe da Estratégia de Saúde da Família. Apesar da importância na organização da AB desde a década de 1990, a regulamentação profissional dos ACS ocorreu somente em 2002, com a promulgação da Lei n.ำ10.507.

O Curso de Cuidador da Pessoa Idosa com Dependência ocorreu em 2009, dentro de uma das diretrizes do Programa de Formação de Profissionais de Nível Médio para a Saúde (PROFAPS), e foi uma das áreas estratégicas para capacitação de trabalhadores, de acordo com 0 estabelecido pela Portaria Ministerial n. 3.189, de 2009 (BRASIL, 2009). Enquanto ETSUS,o CEFOPE/RN participou das ofertas de cursos deste Projeto.

De acordo com Groisman (2013), o Curso de Cuidador da Pessoa Idosa com Dependência integra uma das vertentes do Programa Nacional de Formação de Cuidadores de Idosos (PNFCI), lançado em 2008, que foi potencializado graças ao PROFAPS. A carência de uma formação adequada na área, somada à modificação do perfil demográfico e epidemiológico da população, indicava a necessidade do desenvolvimento de estratégias para minimizar o longo período sem investimento na área, sendo inserida como meta durante o Governo Luiz Inácio Lula da Silva.

Ressalta-se que a oferta deste curso, no estado do Rio Grande do Norte, é relevante. Pois, no último Censo realizado pelo Instituto Brasileiro de Geografia e Estatística (IBGE), observa-se que 9,25\% dos habitantes do RN possuem mais de 60 anos (MELO et al, 2017). Logo, é importante que a sociedade e os serviços de saúde estejam preparados para lidar com esse 
perfil populacional, e o CEFOPE/RN, como espaço de formação, deve contribuir com uma formação na perspectiva do cuidado integral aos sujeitos idosos.

$\mathrm{Na}$ análise, identifica-se que os cursos ofertados pelo CEFOPE/RN no ano de 2010 seguiram a linha política desenvolvida pelo Programa Pacto pela Redução da Mortalidade Infantil Nordeste e Amazônia Legal ${ }^{1}$, lançado pelo Ministério da Saúde e desenvolvido durante os anos de 2009 e 2010.

Dentre as metas a serem alcançadas por esta política, estavam a ampliação da cobertura da Estratégia de Saúde da Família e a qualificação dos profissionais para o acompanhamento das mães (BRASIL, 2010). O documento oficial do Programa apresenta a articulação com as ETSUS para capacitação das equipes que atuam na $A B$. Conforme os achados deste estudo, enquanto ETSUS, o CEFOPE/RN foi envolvido nesse processo.

Assim, frente à caracterização do Pacto pela Redução da Mortalidade Infantil Nordeste e Amazônia Legal, justifica-se o grande quantitativo de cursos com essa temática no CEFOPE/RN no ano de 2010, voltados aos cuidados à mulher, bem como aos cuidados infantil e neonatal, conforme apresentado no Quadro 01, sobre os cursos desenvolvidos.

A análise realizada mostra que a trajetória do CEFOPE/RN se constituiu dentro de um espaço de fortalecimento para os trabalhadores atuantes na área da saúde, e os cursos ofertados demonstram a preocupação com a melhoria da qualidade dos serviços de saúde pública do país. Deste modo, esta pesquisa leva a novos mapeamentos na instituição em estudo, com o intuito de continuar contribuindo com sua trajetória e com a compreensão da formação destinada aos trabalhadores de nível médio em saúde.

Ainda, salienta-se que a década de 1990 é caracterizada como um período no qual o Estado brasileiro estava sendo organizado com diretrizes neoliberais, sendo possível visualizar seus impactos reducionistas não só na área da saúde, mas também na educação, a partir da Lei no 9.394, de 1996, e do Decreto Federal oㅡ 2.208, de 1997. A referida Lei representa uma proposta de desregulamentação compatível a interesses do Estado, ou seja, reduzindo suas obrigações com a oferta de uma educação pública de qualidade (FRIGOTTO; CIAVATTA, 2003).

O Decreto Federal № 2.208, de 1997, por separar o Ensino Médio da Educação Profissional, contribuiu para o fortalecimento da proposta neoliberal do governo Fernando Henrique Cardoso, que potencializou o dualismo estrutural entre classes (SIMÕES, 2010). Destaca-se que esse Decreto foi revogado, em parte, pelo Decreto oㅜ 5.154, de 2004, durante o governo de Luiz Inácio Lula da Silva. Esses regulamentos representam marcos que não retratam conquistas favoráveis à sociedade.

\footnotetext{
${ }^{1}$ Esta política buscou reduzir as desigualdades nas regiões Nordeste e Amazônia Legal, considerando "analfabetismo, da mortalidade infantil, do sub-registro do enfrentamento do baixo dinamismo econômico no meio rural na região" (BRASIL, 2010, p. 7). 
A nova LDB - Lei o 9.394/96 e o Decreto no 2.208/97 vieram desfazer todas as ilusões em torno de uma formação profissional emancipadora. Com a revogação daquele decreto e a aprovação do novo, Decreto no 5.154, reacendem-se as expectativas, mas sempre com um travo de dúvida, em uma sociedade cuja hegemonia está nas leis do lucro do capital (TREIN; CIAVATTA, 2006, p. 110).

Esse fator torna-se mais um desafio a ser enfrentado pelo CEFOPE/RN enquanto ETSUS: garantir a formação de trabalhadores sem aderir a uma formação reducionista e limitada, de acordo com interesses econômicos do capitalismo. Esta afirmação é baseada no cenário apresentado a seguir:

\begin{abstract}
Formar profissionais da saúde não significa, em nenhum nível, a mera adaptação ao existente, com seus fetiches e formas de alienação. Ao invés da informação fragmentada e distorcida, dos fatos e dados parciais, postos fora de contexto, trata-se do trabalho paciente de criticar, mediar, construir uma percepção crítica do profissional de nível médio que trabalha nos serviços de saúde. $O$ que implica conhecimentos teóricos e práticos, culturais e técnicos, qualificando o trabalho e o cidadão no contexto do respeito, da remuneração justa, da participação ativa no cotidiano dos serviços de saúde, na visão crítica qualificada em relação ao país e suas contradições (PEREIRA; RAMOS, 2006, p. 52).
\end{abstract}

A formação de trabalhadores está imersa em uma luta por um projeto de sociedade mais igualitário, que busque romper com questões instáveis no ambiente de trabalho, consolidadas por modelos tradicionais de educação.

Assim, percebe-se que o CEFOPE/RN possui desafios que envolvem a conjuntura nacional da Educação e do Trabalho em Saúde, mesmo tendo como prerrogativas a formação de trabalhadores já inseridos no mundo do trabalho. Na Educação, os desafios ocorrem por seguir os projetos de base nacional que, muitas vezes, seguem correntes redutoras da formação dos sujeitos na Educação Profissional.

Essas correntes, portanto, são pautadas na divisão social do trabalho, e sua presença histórica reduz a forma de inserção de trabalhadores técnicos de saúde, limitando os seus espaços de atuação e os inserindo de maneira precária nos serviços de saúde, vinculados a formações para atuação em cenários diversos que ainda estão se estruturando, mesmo após anos de constituição, o que, consequentemente, interfere nas condições e relações de trabalho.

\title{
4 CONSIDERAÇÕES FINAIS
}

Como resultado deste estudo, tem-se a seguinte reflexão: o CEFOPE/RN desenvolve um importante trabalho para os trabalhadores de nível médio e elementar que atuam no SUS. Os cursos desenvolvidos 
encontram-se alinhados a temas importantes para o fortalecimento do sistema público de saúde e, assim, há a busca da melhoria do atendimento prestado à população que utiliza os serviços públicos de saúde.

A oferta contínua de cursos no período estudado demonstra o trabalho árduo desenvolvido por esta instituição. Todavia, torna-se necessário questionar e acompanhar o desenvolvimento dos programas e projetos demandados pelo Ministério da Saúde. Assim, apesar de serem importantes e trazerem benefícios às ETSUS, os espaços e instituições possuem suas próprias demandas e necessidades. Portanto, deve-se questionar a forma como as políticas de governo ocorrem, em especial, a maneira "verticalizada" com a qual, constantemente, são desenvolvidas.

Reforça-se a necessidade de acompanhamento dos cursos ofertados para que as práticas não sejam induzidas pela concepção apenas instrumental da Educação Profissional em Saúde. Também, o fortalecimento do CEFOPE/RN, enquanto uma das ETSUS, como espaço de luta para o reconhecimento das práticas exercidas por trabalhadores de nível médio e elementar do SUS.

Deste modo, o grande quantitativo de cursos não deve prescindir de métodos de avaliação para que a articulação entre trabalho, educação e saúde esteja baseada na formação crítica e emancipadora daqueles que, apesar do protagonismo no SUS, ainda lutam por reconhecimento em decorrência das precárias condições de trabalho a que estão submetidos.

Este estudo teve como limitação o acesso a informações específicas sobre os cursos, como data de início e descrição, por não estarem descritas nas atas escolares referentes ao período em estudo. Portanto, reflete-se sobre a importância de se ter um sistema de informação acadêmico para que não se percam informações que fragilizem.

Por fim, sugerem-se novos estudos voltados ao corpo docente atuante nos cursos e/ou aos impactos desses cursos nas práticas de trabalho nos serviços de saúde.

\section{REFERÊNCIAS}

BARRÊTTO, M. D. A. Uma trajetória de sucesso na educação profissional em saúde. In: AQUINO, S. N. F. de; MEDEIROS, V. M. R. de (Orgs). A história das instituições de formação e desenvolvimento de servidores públicos do Estado do Rio Grande do Norte. Natal: Escola de Governo, 2010, p. 3546.

BRASIL. Ministério da Saúde. Pacto pela redução da mortalidade infantil no Nordeste e Amazônia Legal: 2009-2010. Brasília: Ministério da Saúde, 2010. 
BRASIL. Ministério da Saúde. Relatório final. Anais... Conferência Nacional de Saúde, Brasília, DF, 2001.

BRASIL. Portaria no 2.970, de 25 de novembro de 2009. Institui a Rede de Escolas Técnicas do SUS (RETSUS) e dispõe sobre as diretrizes para a sua organização. Brasília, 2009.

BRASIL. Portaria no 3.189 , de 18 de dezembro de 2009. Dispõe sobre as diretrizes para a implementação do Programa de Formação de Profissionais de Nível Médio para a Saúde (PROFAPS). Brasília, 2009.

FRIGOTTO, G. O enfoque da dialética materialista histórica na pesquisa educacional. In: Metodologia da pesquisa educacional. 3 ed. São Paulo (SP): Cortez Editora; 1994. p. 69-90.

FRIGOTTO, G.; CIAVATTA, M. Educação básica no Brasil na década de 1990: subordinação ativa e consentida à lógica do mercado. Educ.

Soc., Campinas, v. 24, no 82, p. 93-130, abr. 2003.

GERMANO, R. M. CEFOPE/RN/RN: Uma experiência de formação de recursos humanos em saúde. 1984-1996. Natal: SESAP/RN, 1996.

GHEDIN, E; FRANCO, M. A. S. Questões de método na construção da pesquisa em educação. São Paulo, Cortez, 2011.

GROISMAN D. Formação de Cuidadores de Idosos: avanços e retrocessos na política pública de cuidados no Brasil. In: MOROSINI, M. V.G. C. et al (Orgs). Trabalhadores técnicos em saúde: aspectos da qualificação profissional no SUS. Rio de Janeiro: EPSJV; 2013.

ALMEIDA DE MELO, Laércio et al. Fatores socioeconômicos, demográficos e regionais associados ao envelhecimento populacional. Revista Brasileira de Geriatria e Gerontologia, v. 20, n. 4, 2017.

MINAYO, M. C. S. O desafio do conhecimento: pesquisa qualitativa em saúde. 8. ed. São Paulo: Hucitec, 2013.

MOURA, Dante Henrique. Educação básica e educação profissional e tecnológica: dualidade histórica e perspectivas de integração. Holos, v. 2, p. 4-30, 2007.

PEREIRA, I. B.; RAMOS, M. N. Educação Profissional em saúde. Rio de Janeiro: Editora Fiocruz, 2006.

SILVA, V. O.; SANTANA, P. M. M. A. Curriculum content and Brazilian Health System (SUS): analytical categories, gaps and challenges. Interface (Botucatu). 2015. Disponível em:

http://www.scielosp.org/pdf/icse/v19n52/1807-5762-icse-1807-

576220140017.pdf. Acesso em: out. 2017. 
SIMÕES, C. A. Educação Técnica e escolarização de jovens trabalhadores. In: MOLL, J; FISHER, N. B.; KUENZER, A. (Orgs.). Educação profissional e Tecnológica no Brasil Contemporâneo: Desafios, tensões e possibilidades. CDUed.Porto Alegre: Editora Artmed, 2009, v. , p. 96-120.

TREIN, E.; CIAVATTA, M. A produção capitalista, trabalho e educação: um balanço da discussão nos anos 1980 e 1990. In: FRIGOTTO, G.; CIAVATTA, M. (Orgs.). A formação do cidadão produtivo: a cultura de mercado no ensino médio técnico. Brasília: Ministério da Educação, 2006. 